\title{
AN EFFICIENT METHOD OF GROUP DELAY EQUALIZATION FOR DIGITAL IIR FILTERS
}

\section{Piotr Okoniewski, Jacek Piskorowski}

West Pomeranian University of Technology, Faculty of Electrical Engineering, ul. Sikorskiego 37, 70-313 Szczecin, Poland (\piotr.okoniewski@zut.edu.pl,+48914495221)

\begin{abstract}
The paper presents the equalization problem of non-linear phase response of digital IIR type filters. An improved analytical method of designing a low-order equalizer is presented. The proposed approach is compared with the original method. The genetic algorithm is presented as an iterative method of optimization. The vector and matrix representation of the all-pass equalizer are shown and introduced to the algorithm. The results are compared with the analytical method. In this paper we have also proposed the use of an aging factor and setting the initial population of the genetic algorithm around the solution provided by the analytical methodology.
\end{abstract}

Keywords: IIR filter, digital filter, group delay, phase response, genetic algorithm, equalizer.

(C) 2013 Polish Academy of Sciences. All rights reserved

\section{Introduction}

In the science of measurement, digital signal processing holds a strong position. A transition from measured physical properties to a representation which a computer can understand has been a subject of great interest for many years. Alongside such problems like the accuracy of measurement or the level of influence on the object by the fact of the measurement itself, lies the problem of additional unwanted signals which affects real values. In both analog and digital technologies, the mechanism of filtering is known to be indispensable. Practical knowledge suggests that those unwanted signals are characterized by a certain periodicity. Removing such interference is called filtering in terms of frequency. The designer of digital filters is armed with a vast variety of techniques to successfully accomplish his task. In his knowledge and experience lies the decision which one will be proper for an actual problem. Considering the more popular stationary model, it is crucial to choose between two types of possible designs. The first type of models called finite impulse response (FIR) is stable due to the lack of feedback and can be easily designed to achieve a linear phase response. On the other hand, its mathematical complexity can be significant. The second type of models called infinite impulse response (IIR) preserves the selectivity of the FIR systems with much less complex design. Unfortunately, choosing the IIR system one must remember to check the stability of the design. What is more, the phase response of IIR systems is nonlinear which may cause significant problems even in simple measurement systems.

As a solution to this problem (non-linear phase response) in the continuous-time domain many researchers proposed fine methods including, for example, genetic algorithms [1], adaptive filters [2] or all-pass-based equalization techniques [3]. In this paper we will propose an improved analytical methodology of designing a digital equalizer based on the approach described in [4]. Additionally, the proposed approach and procedures will be introduced to the genetic algorithm in order to improve its robustness in terms of designing the all-pass equalizers. 


\section{Group delay response}

A non-linear phase response of a discrete IIR filter means that the frequencies are delayed by a different amount of time. In signal processing it is often more convenient to use the group delay characteristic. Mathematically, the group delay $\tau(\Omega)$ is defined as a negative derivative of the phase response $\Phi(\Omega)$ with respect to the frequency $\Omega$ :

$$
\tau(\Omega)=-\frac{d(\Phi(\Omega))}{d \Omega} .
$$

As an example we will use a fourth-order low-pass elliptic filter with $60 \mathrm{~Hz}$ cut-off frequency. The pass-band ripple was set to $0.3 \mathrm{~dB}$, the stop band ripple to $40 \mathrm{~dB}$, and the sampling frequency to $1 \mathrm{kHz}$. Fig. 1 presents the magnitude and the group delay responses of this filter.
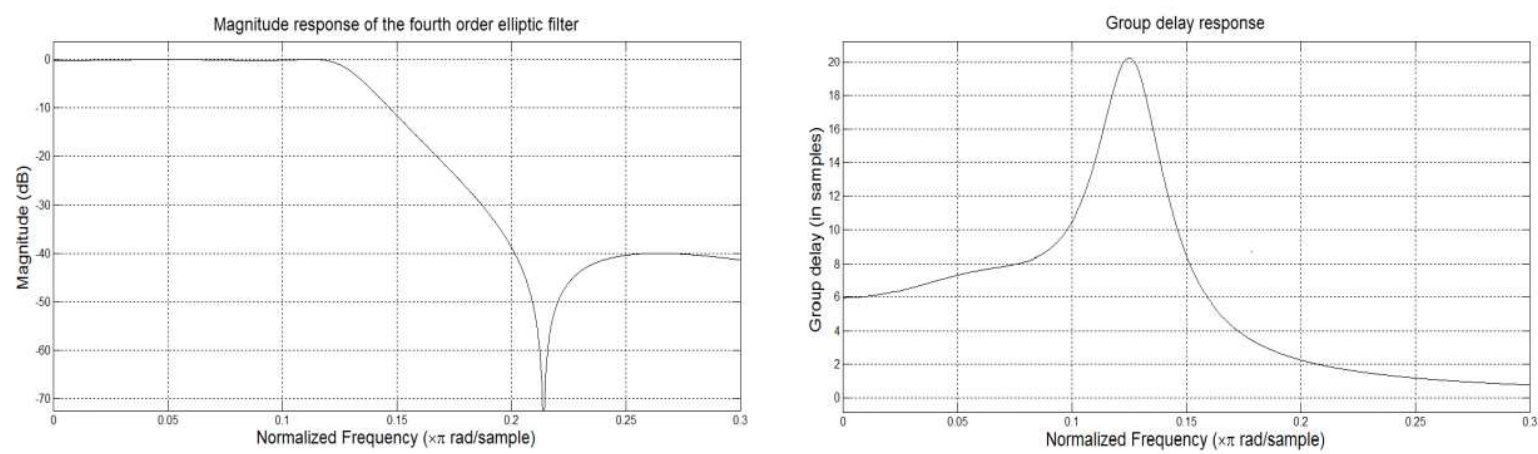

Fig. 1. Magnitude and group delay response of the proposed fourth order elliptic filter.

To show the consequences of a non-constant group delay characteristic, we will introduce to the elliptic filter a testing signal created by the summation of five sinusoidal signals. The first sinusoid is set to $10 \mathrm{~Hz}$, the second to $12 \mathrm{~Hz}, 14 \mathrm{~Hz}$ for the third, $52 \mathrm{~Hz}$ for the fourth, and $58 \mathrm{~Hz}$ for the fifth. The amplitude for all of them is set to 1. Fig. 2 illustrates the testing signal before and after filtering.

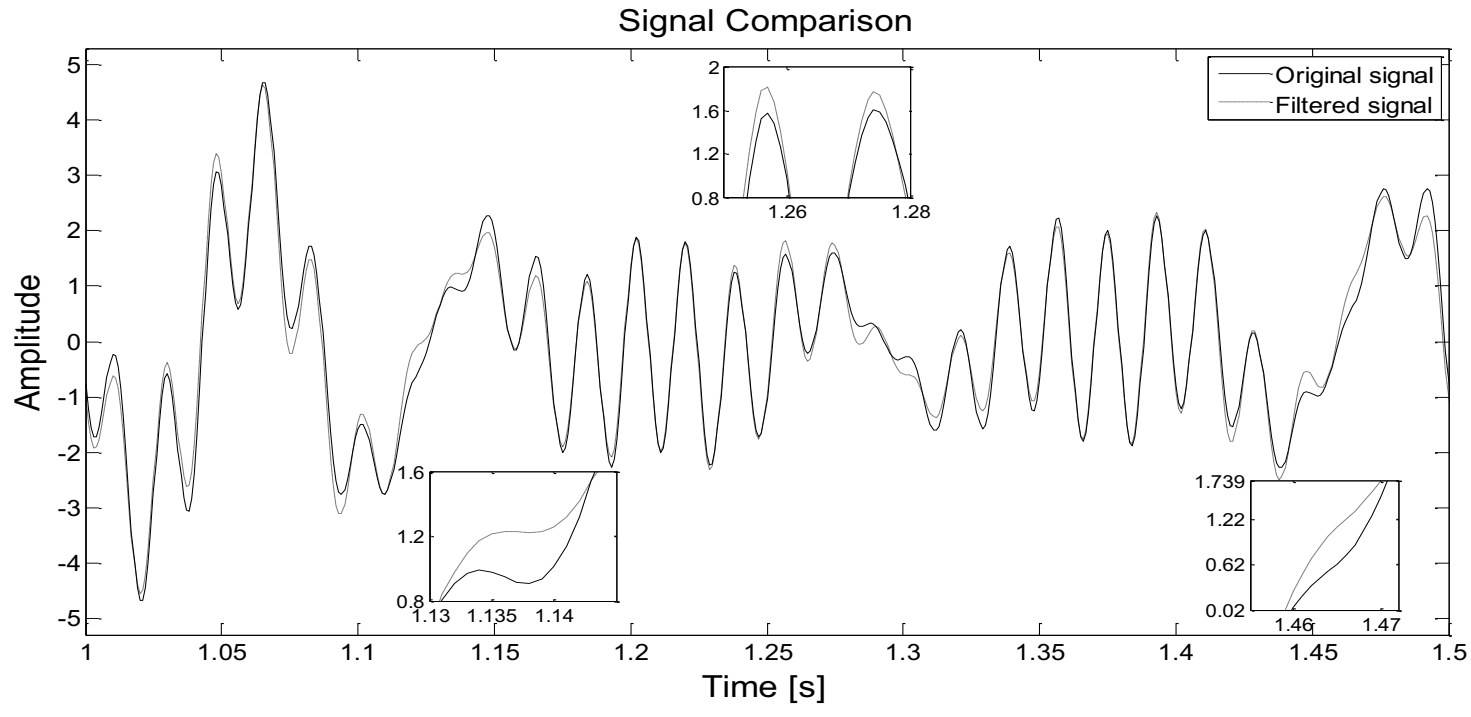

Fig. 2. Comparison of the original signal (solid line) with the filtered one (dashed line).

One needs to notice that all of the frequencies are in the filter's pass-band. The difference in the time between the original and the filtered signal has been neutralized for easier 
comparison. The amplitude losses can be caused by the allowed $0.3 \mathrm{~dB}$ ripple in the passband. Unfortunately, not every difference can be explained by this phenomenon. Some parts of the losses, especially the marked phase shifts and amplifications, are caused by the intersymbol interference (ISI). The ISI effect is a known consequence of the filter's non-linear phase response [5] or similarly, non-constant group delay.

\section{Group delay equalizer}

To counter the effect of non-constant group delay one can find fine methods that were used with continuous-time filters [6-9]. Unfortunately, the proposed approaches cannot be fully put to discrete systems using simple transformations (e.g. bilinear transformation) due to the warping effect in the phase response of the equalized analog filters. In this paper we will focus on designing an all-pass filter which will act as an equalizer of the IIR filter. Fig. 3 presents the block diagram of such a system.

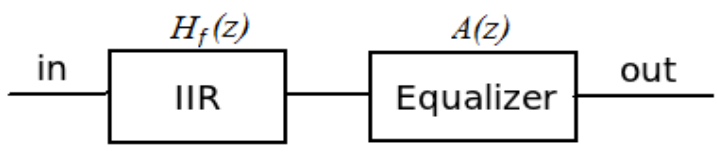

Fig. 3. Equalized IIR filter.

The transfer function of the all-pass filter is defined as:

$$
A(z)=\frac{c_{n}+c_{n-1} z^{-1}+\ldots+z^{-n}}{1+c_{1} z^{-1}+\ldots+c_{n} z^{-n}}
$$

where $c_{n}$ is the $n$-th element of the coefficients vector $\boldsymbol{c}$.

One of the important property of all-pass filters is their constant magnitude response, i.e.

$$
\left|A\left(e^{j \Omega}\right)\right|=1
$$

This means that the magnitude response of the equalized filter will not be affected. On the other hand, the phase response can be reshaped by choosing proper coefficients. Designing the system as in Fig. 3 the following equations become valid:

$$
\begin{gathered}
\left|H_{c}\left(e^{j \Omega}\right)\right|=\left|H_{f}\left(e^{j \Omega}\right)\right| \cdot\left|A\left(e^{j \Omega}\right)\right|=\left|H_{f}\left(e^{j \Omega}\right)\right|, \\
\tau_{c}(\Omega)=\tau_{f}(\Omega)+\tau_{a}(\Omega),
\end{gathered}
$$

where $H_{c}, \tau_{c}$ refer to the whole system, $H_{f}, \tau_{f}$ to the original filter, and $A, \tau_{a}$ describe the designed equalizer. Equation (4) means that the final magnitude response will not be affected by adding the equalizer and will preserve the shape of the magnitude response of the original IIR filter. Equation (5) means that the final group delay is a summation of group delays of both filters. 


\section{Pole placement method}

By choosing a specific vector $c$ the poles and zeros of the all-pass prototype are calculated. According to (4) and (5) only the group delay response can be modified. The problem of efficient equalization lies in selecting such placement of the poles and zeros that the final characteristic of the group delay is acceptable. In our studies we concentrated on providing a characteristic with the lowest difference between the maximum and minimum value of the group delay in the equalized filter's pass-band. In [4] and [10] Petraglia and Quelhas approached this problem by combining analytical analysis with iterative optimization techniques. Our studies showed that in terms of low order (up to 8-th) analytical equalization their strategy can be improved by choosing a different methodology of calculating the all-pass equalizer.

The main idea of the method proposed in $[4,10]$ was to analyze an equalized IIR filter on the $z$-plane. The outermost pair of poles was considered in terms of their angle and radius. Then, according to specific strategies, the poles of the all-pass equalizer were calculated. Fig. 5 presents a graphical idea of the method.

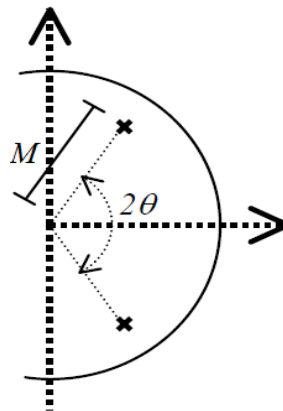

(a)

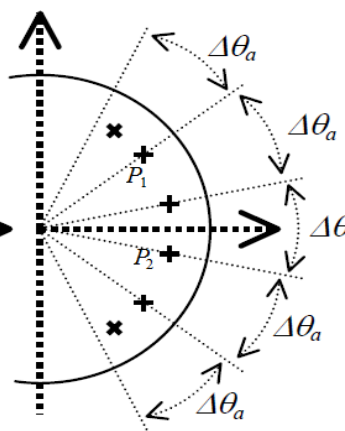

(b)

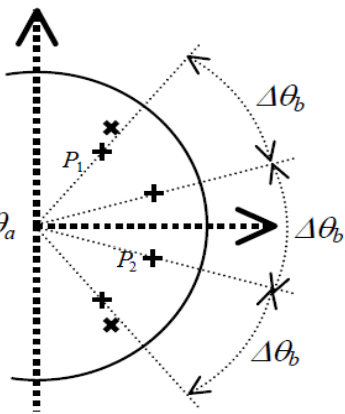

(c)

Fig. 4. Magnitude and angle between conjugated poles (a). Two strategies proposed in [4] (b), (c); Poles marked by " $\mathrm{x}$ " are the outermost poles (conjugated) of the original IIR filter; The poles marked by "+" are the poles of the equalizer.

In our studies we come up with a new methodology of the pole placement that improves final group delay in terms of the difference between maximum and minimum values in the pass-band. We propose to alter the equations delivered by [4] and [10] with our solutions (6) (9) and (11)-(14). The specific values of the coefficients were chosen based on the experimental tests and careful analysis of the all-pass filter behavior with different pole placement. As an initial approach the all-pass equalizer is calculated according to the following strategy:

$$
\Delta \theta_{a}=\frac{2.23 \theta}{2 n+1},
$$

where $\Delta \theta_{a}$ is the angle between two conjugated poles (first strategy), $n$ is the number of the second-order all-pass sections of the equalizer, and $\theta$ is the angle of the equalized filter outermost pole.

Starting from the outermost pole, the magnitudes of the first and second pair of poles are as follows:

$$
\begin{gathered}
P(1)=1.8 M-0.812, \\
P(2)=1.2 P(1)-0.211,
\end{gathered}
$$

where $P$ are the specific conjugated poles of the all-pass equalizer and $M$ is the magnitude value of the equalized filter outermost pole. 
For the third and fourth pair of poles the magnitude can be expressed in the following form:

$$
P(q)=0.97 P(q-1)+0.032-0.0025 n,
$$

where $q=[3,4]$ is the third and the fourth pair of conjugated poles.

Then, according to [4], the influence of the filter original group delay on the final response must be verified using the following relationship:

$$
1.07^{n+1} \tau_{a, \theta}<0.85 \cdot 1.04^{n} \tau_{f, \max },
$$

where $\tau_{a, \theta}$ is the group delay value of the equalizer evaluated at the cutoff frequency and $\tau_{f, \max }$ is the maximum value of the group delay of the equalized filter in the pass-band.

If inequality (10) is not fulfilled then the poles of the all-pass filter must be calculated according to the following strategy:

$$
\Delta \theta_{b}=\frac{0.8 \theta+0.0013}{2 n-1}
$$

where $\Delta \theta_{b}$ is the angle between two conjugated poles (second strategy).

Again, the magnitudes of the first and second pair of poles are as follows:

$$
\begin{aligned}
& P(1)=1.8 M-0.805, \\
& P(2)=1.2 P(1)-0.2,
\end{aligned}
$$

and for the third and fourth pair of poles:

$$
P(q)=0.97 P(q-1)+0.03-0.0028 n .
$$

One must remember that the presented method is an analytical approach used to solve a complicated problem. Therefore, the obtained results should be doubly checked. As for the stability of the whole combined system, the method ensures stability as long as the equalized filter is stable.

\subsection{Simulations}

The proposed methodology of analytical equalization was tested on various examples of elliptic, Butterworth, and Chebyshev filters. Fig. 5 depicts the group delay response of the fourth-order elliptic filter mentioned in Sec. 2 equalized by means of the original methodology proposed in [4] and the one presented in this paper.

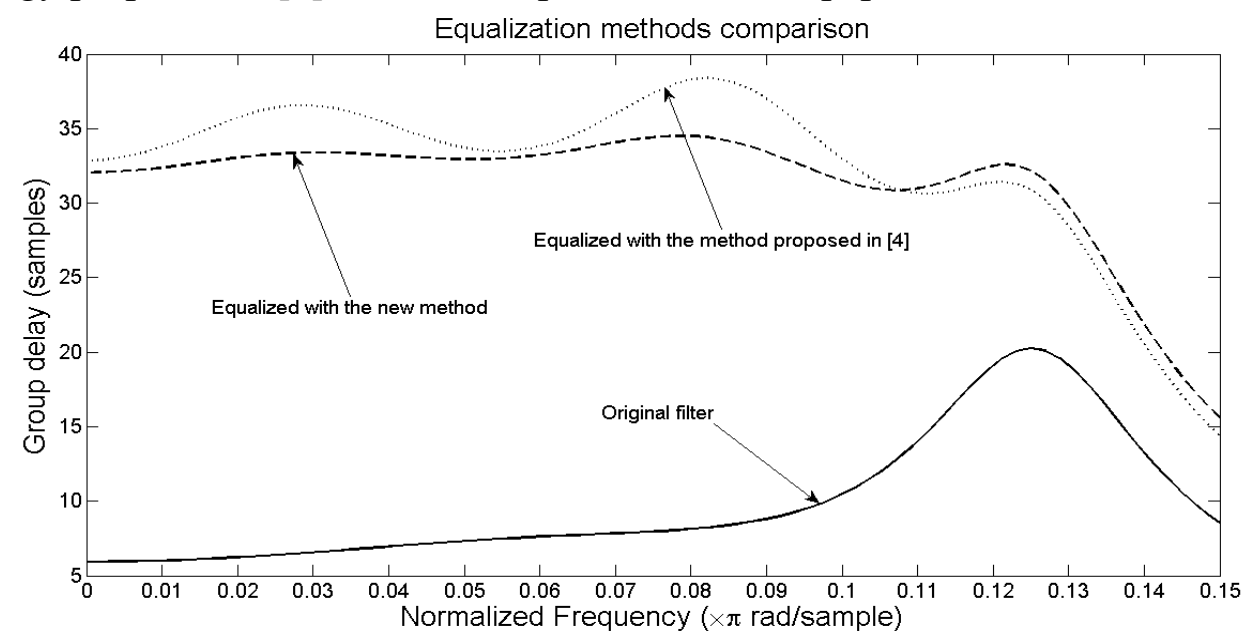

Fig. 5. Comparison of the presented method of analytical equalization with the analytical part proposed in [4]. 
As one can notice, using the proposed method, the difference between the maximum and minimum value of the group delay is significantly decreased. Specific values along with other examples of both compared methods can be found in Table 1. The order of the equalizer was chosen arbitrarily to ensure a practical solution.

Table 1. Simulation results of the presented method of equalizing various IIR type filters.

\begin{tabular}{|c|c|c|c|}
\hline Analyzed filter & $\operatorname{Max}\left(\tau_{c}\right)$ & $\operatorname{Min}\left(\tau_{c}\right)$ & $\Delta \tau_{c p}$ \\
\hline $\begin{array}{c}\text { Elliptic; } 4^{\text {th }} \text {-order } \omega_{C}=0.12 ; R_{p}=0.3 \mathrm{~dB} \\
R_{S}=40 \mathrm{~dB} ; \tau_{d}=13.32 ; N_{a}=4\end{array}$ & $\mathbf{3 4 . 5 1}(38.36)$ & $\mathbf{3 0 . 8 4}(30.65)$ & $3.67(7.71)$ \\
\hline $\begin{array}{c}\text { Elliptic; } 3^{\mathrm{rd}} \text {-order; } \omega_{c}=0.3 ; R_{p}=1 \mathrm{~dB} \\
\qquad R_{S}=30 \mathrm{~dB} ; \tau_{d}=4.72 ; N_{a}=8\end{array}$ & $21.71(24.05)$ & $\mathbf{2 0 . 0 9}(21)$ & $1.62(3.05)$ \\
\hline $\begin{array}{c}\text { Elliptic; } 5^{\text {th }} \text {-order; } \omega_{C}=0.1 ; R_{p}=0.2 \mathrm{~dB} \\
\qquad R_{S}=70 \mathrm{~dB} ; \tau_{d}=18.65 ; N_{a}=8\end{array}$ & $75.23(75.62)$ & $66.72(63.4)$ & $8.51(12.22)$ \\
\hline $\begin{array}{l}\text { Chebyshev I; } 4 \text { th-order; } \omega_{C}=0.17 \\
R_{p}=1.3 \mathrm{~dB} ; \tau_{d}=12.42 ; N_{a}=6\end{array}$ & $36.79(38.23)$ & $33.56(29.13)$ & $3.23(9.1)$ \\
\hline $\begin{array}{c}\text { Chebyshev I; } 4^{\text {th }} \text {-order; } \omega_{C}=0.27 \\
R_{p}=0.9 \mathrm{~dB} ; \tau_{d}=7.46 ; N_{a}=8\end{array}$ & $27.33(29.73)$ & $25.65(25.26)$ & $1.68(4.47)$ \\
\hline Butterworth; 3rd-order; $\omega_{c}=0.2 ; \tau_{d}=1.45 ; N_{a}=4$ & $12.64(15.85)$ & $\mathbf{1 1 . 5 3}(13.1)$ & $1.11(2.75)$ \\
\hline Butterworth; $5^{\text {th }}$-order; $\omega_{C}=0.32 ; \tau_{d}=3.01 ; N_{a}=6$ & $15.19(17.28)$ & $15.02(15.75)$ & $\mathbf{0 . 1 7}(1.53)$ \\
\hline
\end{tabular}

The nomenclature in Table 1 is as follows: $\omega_{c}$ is the normalized cutoff frequency, $R_{p}$ is the allowed ripple in the pass-band, $R_{s}$ is the demanded ripple in the stop-band, $\tau_{d}$ is the difference between the maximum and minimum values of the original group delay in the filters pass-band, $\Delta \tau_{c p}$ is the difference between the maximum and minimum value of the equalized group delay in the filters pass-band, $N_{a}$ is the order of the all-pass equalizer. The values in brackets are calculated using the analytical part of the method proposed in [4].

As one can notice, the proposed analytical method of compensation lowers the difference between maximum and minimum values of the group delay which is very important from the signal-processing point of view. Fig. 6 presents another example of equalization of the $5^{\text {th }}$ order Butterworth filter $\left(\omega_{c}=0.32\right)$ with the 6th-order all-pass equalizer.

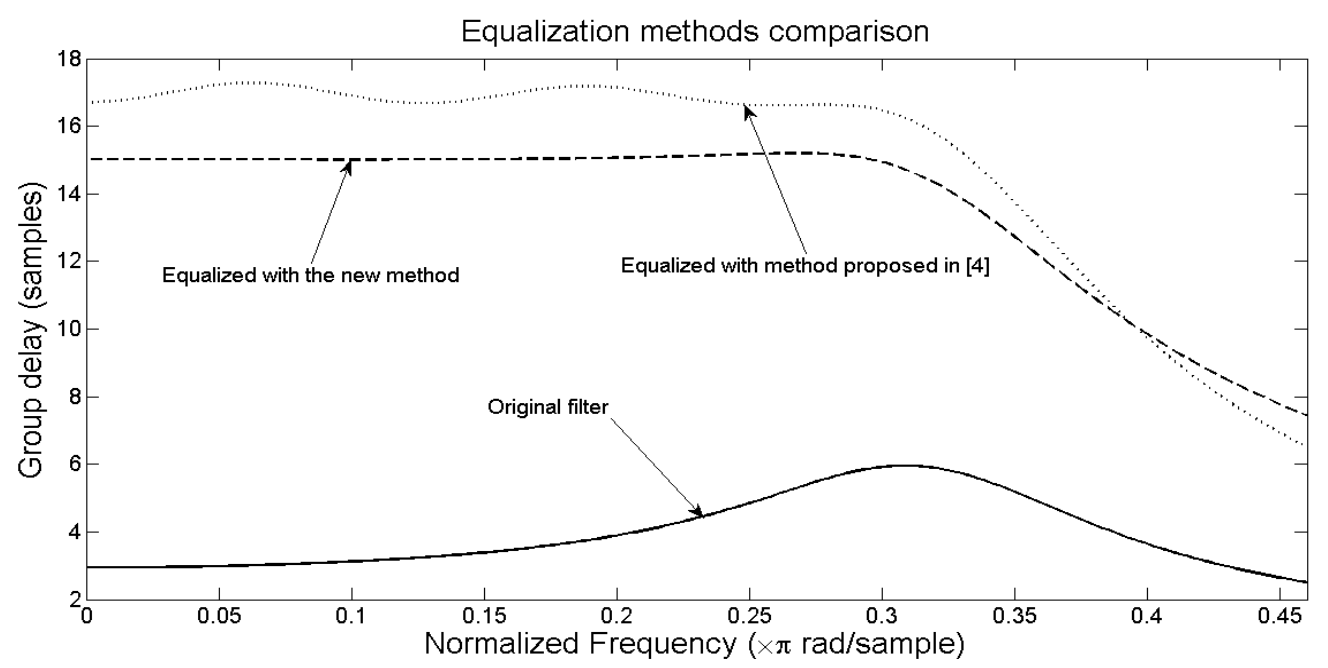

Fig. 6. Comparison of the presented method with the one proposed in [4] for the $5^{\text {th }}$-order Butterworth filter. 
An analytical equalization unfortunately does not provide an optimal solution. Due to the nature of the equalization problem and the need to achieve an as flat as possible characteristic of the group delay, one might need to turn to iterative methods of compensation. There are fine techniques of optimizing the placement of the poles but the higher the order of the equalizer, the higher the risk of ending in one of the local minima. In our studies we proposed a technique based on genetic algorithm with new approaches regarding the use of matrix representation of the optimized all-pass filter.

\section{Genetic algorithm}

Evolutionary algorithms (EA) gained a strong position in optimization theory. Genetic algorithms (GA) are the most popular among the EA. They are widely used thanks to their natural and intuitive procedures. Concisely stated, a genetic algorithm is an attempt to copy natural and biological aspects of evolution expressed in a chosen programming language [11], [12].

Given an optimization problem to solve, the GA is introduced with a set of generated solutions (population of candidates) and a fitness function. The first set of solutions usually is generated randomly, but it can also be fixed with a priori knowledge to focus on a specific area. The fitness function is a metric which can evaluate each candidate in a quantitative manner. Considering a randomly generated population, some of the candidates may not work in terms of stated problem boundaries. Those candidates must be deleted as they host no hope of finding useful solution. The candidates are reproduced to form a second-generation population. The process of reproduction can be characterized with a variety of techniques in terms of choosing the right candidate itself and the changes it can undergo. To introduce a small rate of variety to the population, a mutation process can be conducted to the newly generated unit. The process now repeats itself for several hundreds or thousands generations. The candidate which will be at the top of the final generation (due to generations limit or no significant improvement) can be considered as the optimal solution in terms of a given fitness function [13]. Fig. 7 illustrates a simplified graphical representation of the GA.

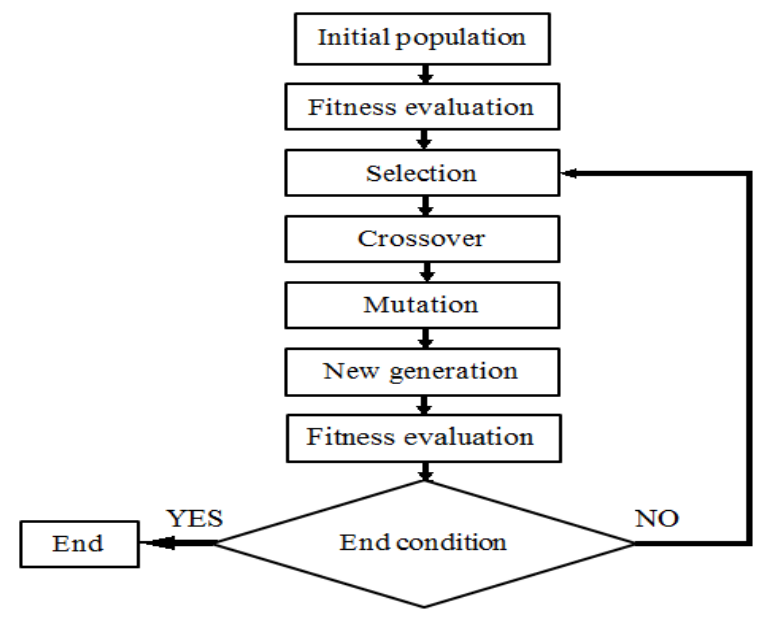

Fig. 7. Simplified graphical representation of the genetic algorithm.

Analyzing the way the genetic algorithm works, one can easily point out a few important strengths of this method. First of all the computation is parallel in terms of searching through the solutions space. Most methods working in a serial manner must abandon all the previous work if the algorithm returned only a local extremum, for example because of the poorly selected starting point. Following that, the fitness function can be more complex, i.e. may 
have many local extrema or discontinuities [14]. Another quality which is extremely valuable is that the GAs are very efficient in terms of solving multivariable problems [15].

Of course, as any other optimization method, genetic algorithms are not free from limitations. For example the performance of the algorithm strongly depends on the fitness function. If the problem is complicated it might by difficult to write a proper function ensuring the outcome will be in the expected manner [16]. Additionally, for a practical reason, one must decide about the size of population, crossing techniques and the generations number to maintain a reasonable time of computation.

\subsection{Equalizer design using Gas}

The position of each pole and zero has influence on the group delay value in every part of the characteristic. In other words, one can imagine that by changing the magnitude or angle of even one pair of poles, the whole group delay is affected. Analyzing the complexity of the problem of minimizing the difference between the maximum and minimum value of the group delay, the potential solution space is expected to be rich with local minima. The genetic algorithm due to its ability to avoid those minima can be a very powerful tool in terms of designing or optimizing a proper equalizer.

Similarly as in Sec. 3, let us consider the all-pass filter given by (2) as an equalizer prototype. One of possible representations of such prototype are the pairs of angles and magnitudes for each pole and zero on the $z$-plane. To create the initial population for the genetic algorithm, one must characterize a single candidate. Due to the need of maintaining constant magnitude response given by (3) of the all-pass filter, the zeros can be calculated on the basis of the pole placement. As the pairs of the poles are conjugated, it is only crucial to know the angle and magnitude of the one pole (of each pair) to calculate the other. By that fact, to reduce the complexity of each candidate while still being able to rebuild the all-pass filter, we consider only the angles and magnitudes of one pole of each pair. Fig. 8 depicts one of the candidate's possible vector representation of the all-pass equalizer prototype.

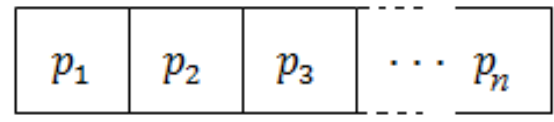

Fig. 8. First candidate representation where $p_{n}$ is the $n$-th pole of the all-pass prototype.

As mentioned before, one unfortunately placed pole can affect the performance of the whole prototype. By that fact, we proposed a second possible representation which allows to introduce additional crossover techniques. Fig. 9 depicts a matrix design of such candidate.

\begin{tabular}{|c|c|c|c|}
\hline$M_{1}$ & $M_{2}$ & $M_{3}$ & $\ldots$ \\
\hline$\theta_{1}$ & $\theta_{2}$ & $\theta_{3}$ & $\ldots$
\end{tabular} $\bar{M}_{n}$

Fig. 9. Second candidate representation where $M_{n}=\left|p_{n}\right|$ and $\theta_{n}=\angle\left(p_{n}\right)$.

The crossover process is usually characterized by three standard techniques: single point crossover, two point crossover, and uniform crossover. These techniques are responsible for switching the poles between two candidates (vector representation) in order to acquire another generation. In the case of the second, the matrix representation, we developed a crossing method preserving the ability to switch the poles as a whole (column) or just one of the poles 
value. Additionally, such approach allows to perform the mentioned standard techniques at rows affecting only the magnitude or angle set.

The main intention of such an approach is to avoid eliminating the candidate with promising poles placement, unfortunately evaluated very poorly by the fitness function due to one single faulty value.

The mutation process, naturally with a small chance of occurrence, changes one of the candidates feature. In the case of the first representation, a single pole can be randomly placed within the chosen boundaries. This means that the mutation will probably affect both the magnitude and angle of such pole. The second representation allows to preserve one of the pole values, e.g. the angle, while the magnitude can have random changes.

During our studies we also introduced an aging factor (expressed as a percentage value) to the mutation probability and allowed boundaries. The main idea was to apply a larger diversity to the population if there is no improvement for a significant amount of generations. The aging factor is defined as follows:

$$
\text { aging factor }=0.1 \cdot e^{k / 19} \text {, }
$$

where $k$ is the number of generations since the last overall improvement. Fig. 10 depicts the proposed aging factor as a function of the number of generations $k$.

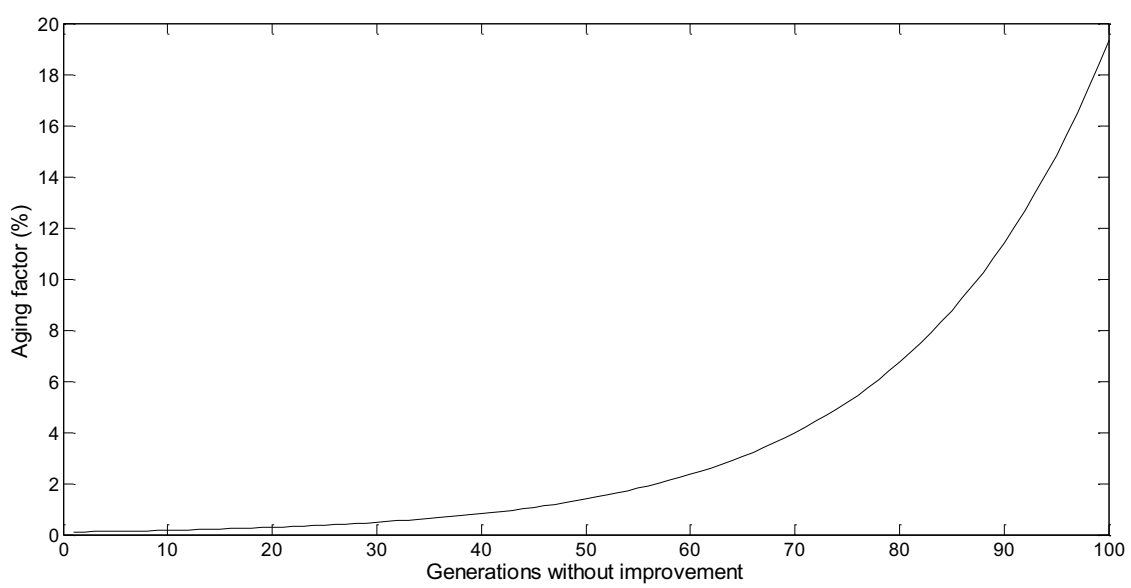

Fig. 10. Proposed aging factor on the span of 100 generations without improvement.

Assuming the initial mutation rate at $1 \%$, the $2 \%$ rate will be achieved after 45 generations without improvement. After 100 generations the mutation probability will reach $20.31 \%$. Of course if the best solution improves, the aging factor resets itself.

\subsection{Simulations}

One of the often discussed problem concerning genetic algorithms is a proper choice of the population size and the rates of possible crossover and mutation. A population too large will demand a more powerful processing unit to deliver a solution in a reasonable time. On the other hand, a too small population might not be able to solve a complicated problem at all. The fitness function evaluating each candidate was chosen as a difference between the maximum and minimum value of the group delay characteristic in the filters pass-band. During our experiments a half of the best population is always kept and allowed to reproduce. The selection for the crossover of the remaining candidates is conducted on the basis of the equally spaced roulette-wheel technique. This means that all of the candidates have the same chance to take part in the mating process restricted only by the probability factor (crossover 
rate). As for the crossover and mutation rates, scientists report respectively $50 \%-100 \%$ for the crossover and $0.5 \%-1 \%$ for the mutation rate as the most promising values [17], [18].

Fig. 11 presents the group delay response of the fourth-order elliptic filter (mentioned in Sec. 2) equalized with the all-pass prototype designed using the genetic algorithm approach. The fitness function aimed at achieving the lowest difference between maximum and minimum group delay value in the pass-band.

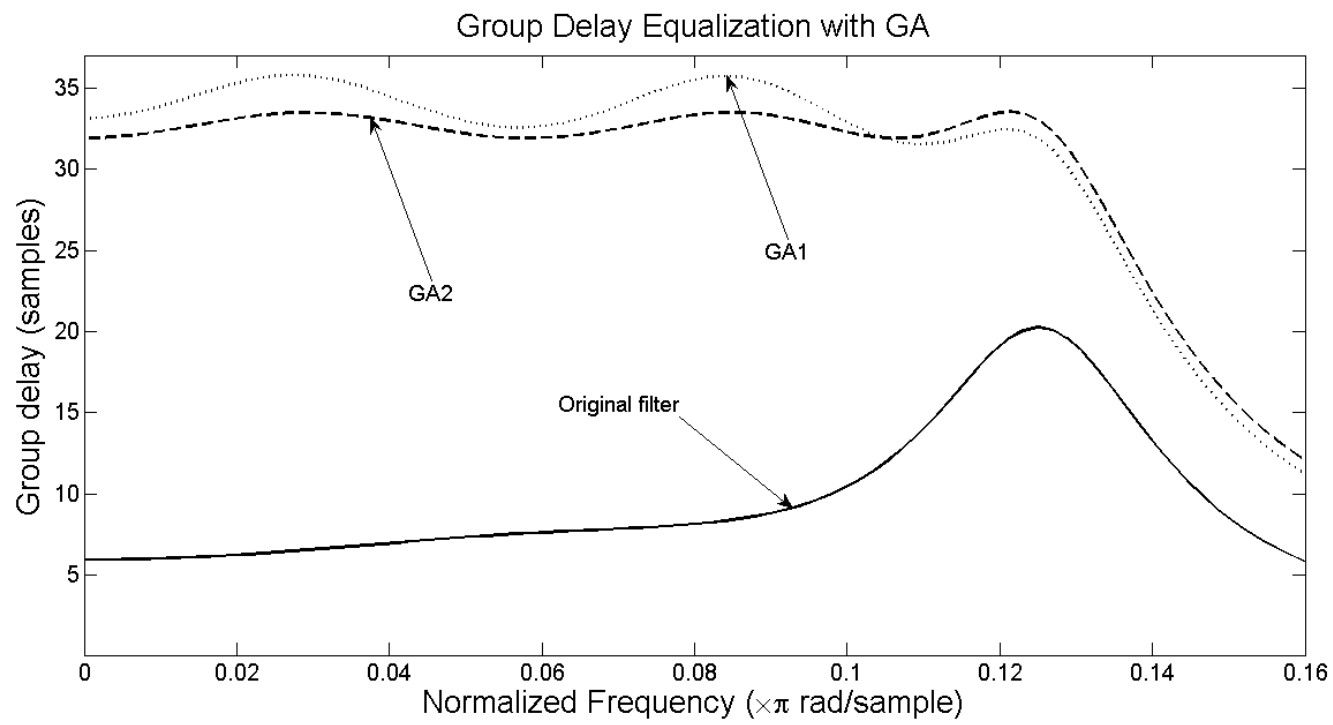

Fig. 11. Group delay compensation with the use of the genetic algorithm.

The GA1 final group delay characteristic was achieved with the use of the pole representation (Fig. 8). The GA2 characteristic was achieved by designing the equalizer with the second representation, i.e. the magnitude and angle representation of the prototype (Fig. 9). In Table 2 the values of the difference between maximum and minimum of the group delay in the passband are pointed out along with the number of the computed generations.

Table 2. Simulation results of the group delay equalization with the use of the genetic algorithm

(Random population size $=60$; Crossover rate $=90 \%$; Mutation rate $=1 \%$; Without aging factor).

\begin{tabular}{|c|c|c|c|c|}
\hline Analyzed filter & $\Delta \tau_{c p} \mathrm{GA} 1$ & GA1 gen. & $\Delta \tau_{c p} \mathrm{GA} 2$ & GA2 gen. \\
\hline $\begin{array}{c}\text { Elliptic; } 4 \text { th-order; } \omega_{C}=0.12 ; R_{p}=0.3 \mathrm{~dB} ; \\
R_{S}=40 \mathrm{~dB} ; \tau_{d}=13.32 ; N_{a}=4\end{array}$ & 3.5 & 649 & 1.57 & 527 \\
\hline $\begin{array}{c}\text { Elliptic; } 3^{\text {rd }} \text {-order; } \omega_{C}=0.3 ; R_{p}=1 \mathrm{~dB} ; \\
R_{S}=30 \mathrm{~dB} ; \tau_{d}=4.72 ; N_{a}=8\end{array}$ & 1.29 & 711 & 0.05 & 601 \\
\hline $\begin{array}{c}\text { Elliptic; } 5^{\text {th }} \text {-order; } \omega_{C}=0.1 ; R_{p}=0.2 \mathrm{~dB} ; \\
R_{S}=70 \mathrm{~dB} ; \tau_{d}=18.65 ; N_{a}=8\end{array}$ & 0.58 & 628 & 0.21 & 599 \\
\hline $\begin{array}{c}\text { Chebyshev I; } 4^{\text {th }} \text {-order; } \omega_{C}=0.17 ; \\
R_{p}=1.3 \mathrm{~dB} ; \tau_{d}=12.42 ; N_{a}=6\end{array}$ & 2.09 & 589 & 2.09 & 506 \\
\hline $\begin{array}{c}\text { Chebyshev I; } 4^{\text {th }} \text {-order; } \omega_{C}=0.27 ; \\
R_{p}=0.9 \mathrm{~dB} ; \tau_{d}=7.46 ; N_{a}=8\end{array}$ & 1.01 & 587 & 0.97 & 540 \\
\hline Butterworth; $3^{\text {rd }}$-order; $\omega_{C}=0.2 ; \tau_{d}=1.45 ; N_{a}=4$ & 0.08 & 140 & 0.07 & 70 \\
\hline Butterworth; $5^{\text {th }}$-order; $\omega_{C}=0.32 ; \tau_{d}=3.01 ; N_{a}=6$ & 0.08 & 133 & 0.05 & 82 \\
\hline
\end{tabular}

Both simulations started with randomly generated sets of populations. The stop condition was set to 100 generations without improvement. As one can notice, the iterative (GA) method of 
compensation reached a better performance in terms of designing a proper equalizer than the analytical method presented in Sec. 4. Unfortunately, such performance is occupied by a quite significant number of needed iterations to provide such solution. What is also worth mentioning, the second representation of the all-pass prototype managed to achieve equally or slightly better performance in both the final group delay difference and needed generations.

As a second part of our studies on the GA, we concentrated on increasing the robustness of the computation. We achieved that by introducing to the algorithm the aging factor mentioned earlier. We also fixed the initial population around the analytical solution provided by the improved method described in Sec. 4. Table 3 presents the improvement of the robustness of the GA in the case of magnitude and angle representation as, during our studies, it proved to be superior.

Table 3. Simulation results of the group delay equalization with the use of the genetic algorithm (Fixed population size $=60 ;$ Crossover rate $=90 \%$; Mutation rate $=1 \%$; With aging factor)

\begin{tabular}{|c|c|c|}
\hline Analyzed filter & $\Delta \tau_{c p}$ GA2 & GA2 gen. \\
\hline $\begin{array}{c}\text { Elliptic; } 4^{\text {th }} \text {-order; } \omega_{\mathcal{C}}=0.12 ; R_{p}=0.3 \mathrm{~dB} ; \\
R_{S}=40 \mathrm{~dB} ; \tau_{d}=13.32 ; N_{a}=4\end{array}$ & $\mathbf{2 3 8}(527)$ \\
\hline $\begin{array}{c}\text { Elliptic; } 3^{\text {rd }} \text {-order; } \omega_{\mathcal{C}}=0.3 ; R_{p}=1 \mathrm{~dB} ; \\
R_{S}=30 \mathrm{~dB} ; \tau_{d}=4.72 ; N_{a}=8\end{array}$ & 0.05 & $\mathbf{2 2 0}(601)$ \\
\hline $\begin{array}{c}\text { Elliptic} ; 5 \text { th-order; } \omega_{\mathcal{C}}=0.1 ; R_{p}=0.2 \mathrm{~dB} ; \\
R_{S}=70 \mathrm{~dB} ; \tau_{d}=18.65 ; N_{a}=8\end{array}$ & 0.21 & $\mathbf{2 0 2}(599)$ \\
\hline $\begin{array}{c}\text { Chebyshev I; } 4^{\text {th }} \text {-order; } \omega_{\mathcal{C}}=0.17 ; \\
R_{p}=1.3 \mathrm{~dB} ; \tau_{d}=12.42 ; N_{a}=6\end{array}$ & 2.08 & $\mathbf{1 8 9}(506)$ \\
\hline $\begin{array}{c}\text { Chebyshev I; } 4^{\text {th }} \text {-order; } \omega_{\mathcal{C}}=0.27 ; \\
R_{p}=0.9 \mathrm{~dB} ; \tau_{d}=7.46 ; N_{a}=8\end{array}$ & 0.97 & $\mathbf{1 7 7}(540)$ \\
\hline Butterworth; 3 rd-order; $\omega_{\mathcal{C}}=0.2 ; \tau_{d}=1.45 ; N_{a}=4$ & 0.07 & $\mathbf{5 1}(70)$ \\
\hline Butterworth; $5^{\text {th }}$-order; $\omega_{\mathcal{C}}=0.32 ; \tau_{d}=3.01 ; N_{a}=6$ & 0.05 & $\mathbf{6 6}(82)$ \\
\hline
\end{tabular}

As one can notice, the generations number needed to compute the solution was significantly reduced (earlier values in brackets).

\section{Conclusions}

The non-constant group delay characteristic of IIR filters can be responsible for the unwanted amplitude losses. From the metrological point of view such losses are added to the total error of the measurement procedure and can be significant even in simple systems. To eliminate this problem we have proposed an all-pass filter as an equalizer. Our studies aimed at achieving the lowest difference between the maximum and minimum value of the group delay characteristic in the filters pass-band. As the analytical method of finding proper coefficients, we have improved a known solution based on the pole placement of the original filter. The method was tested on various digital filters and the results were presented.

As the iterative method we have used the genetic algorithm to compute optimal coefficients. We have proposed two representations of the all-pass prototype: the pole representation and the magnitude-angle matrix representation. To compare the iterative method with the analytical one, we have presented the results for the same sets of examples. To improve the robustness of the genetic algorithm we have introduced the aging factor to increase the mutation rate and boundaries if there is no improvement over generations. We 
have also presented the results when the initial population is set around the solution calculated by the proposed analytical solution. Combining the iterative method with the initial condition provided by the analytical solution proved to be an efficient tool to compensate the nonconstant group delay characteristic of the IIR type filters.

\section{Acknowledgements}

This work has been supported by the Ministry of Science and Higher Education of the Republic of Poland under grant contract N N505 484740.

\section{References}

[1] Ziska P., Vrbata J. (2006). Method for design of analog group delay equalizers. Proc. IEEE ISCAS, 445-448.

[2] Farhang-Boroujeny B., Nooshfar S. (1991). Adaptive phase equalization using all-pass filters. Proc. IEEE ICC, $1403-1407$.

[3] Umino K., Andersen J., Hove R.G. (1990). A novel IIR filter delay equalizer design approach using a personal computer. Proc. IEEE ISCAS, 1,137-140.

[4] Quelhas M.F., Petraglia A. (2003). Group delay equalization of discrete time filters. Proc. IEEE ISIE, 2, 924- 927.

[5] Zhang L., Kwasniewski T. (2010). Optimal equalization for reducing the impact of channel group delay distortion on high-speed backplane data transmission. Int. J. of Electron. and Commun., 64(7), 671-681.

[6] Piskorowski J., Kaszynski R., Gutierrez de Anda M. A., Sarmiento-Reyes A. (2008) Group delay compensation and settling time minimization in continuous-time elliptic filters. Proc. IEEE MELECON, 12-16.

[7] Gupta A., Parsa A., et al., (2010). Group-delay engineered noncommensurate transmission line all-pass network for analog signal processing. IEEE Trans. Microw. Theory Tech., 58(9), 2392-2407.

[8] Piskorowski J., Gutierrez de Anda M. A. (2009). A New Class of Continuous-Time Delay-Compensated Parameter-Varying Low-Pass Elliptic Filters With Improved Dynamic Behavior. IEEE Trans. Circuits Syst. I, Reg. Papers. 56(1), 179-189.

[9] Piskorowski J. (2006). Phase-Compensated Time-Varying Butterworth Filters. Analog Integr. Circuits Signal Process., 47(2), 233-241.

[10] Quelhas M.F., Petraglia A. (2005). Initial solution for the optimum design delay equalizers. Proc. IEEE ISCAS, 4, 3587-3590.

[11] Golonek T., Jantos P., Rutkowski J., (2012). Stimulus with limited band optimization for analogue circuit testing. Metrol. Meas. Syst., 19(1), 73-84.

[12] Goldberg D.E., (1989). Genetic Algorithms in Search, Optimization, and Machine Learning. Boston: Addison Wesley.

[13] Holland J. (1992). Genetic algorithms. Scientific American. 66-72.

[14] C. Coello (June 2005). An updated survey of GA-based multiobjective optimization techniques. $A C M$ Computing Surveys, 32(2), 109-143.

[15] Bird J., Layzell P. (2002). The evolved radio and its implications for modeling the evolution of novel sensors. Proceedings of the 2002 Congress on Evolutionary Computation. 1836-1841.

[16] Srinivas, M., Patnaik, L.M. (1994). Adaptive probabilities of crossover and mutation in genetic algorithms. IEEE Trans. Syst., Man, Cybern., Syst., 24(4), 656-667.

[17] Haupt, R.L. (2000). Optimum population size and mutation rate for a simple real genetic algorithm that optimizes array factors. IEEE APS., 2(2), 1034-1037. 